\title{
Mass Bounds on Light and Heavy Neutrinos from Radiative Minimal-Flavor-Violation Leptogenesis
}

DOI:

10.1103/PhysRevD.92.085016

\section{Document Version}

Final published version

Link to publication record in Manchester Research Explorer

\section{Citation for published version (APA):}

Pilaftsis, A., \& Teresi, D. (2015). Mass Bounds on Light and Heavy Neutrinos from Radiative Minimal-FlavorViolation Leptogenesis. Physical Review D (Particles, Fields, Gravitation and Cosmology), 92(8), 085016. https://doi.org/10.1103/PhysRevD.92.085016

\section{Published in:}

Physical Review D (Particles, Fields, Gravitation and Cosmology)

\section{Citing this paper}

Please note that where the full-text provided on Manchester Research Explorer is the Author Accepted Manuscript or Proof version this may differ from the final Published version. If citing, it is advised that you check and use the publisher's definitive version.

\section{General rights}

Copyright and moral rights for the publications made accessible in the Research Explorer are retained by the authors and/or other copyright owners and it is a condition of accessing publications that users recognise and abide by the legal requirements associated with these rights.

\section{Takedown policy}

If you believe that this document breaches copyright please refer to the University of Manchester's Takedown Procedures [http://man.ac.uk/04Y6Bo] or contact uml.scholarlycommunications@manchester.ac.uk providing relevant details, so we can investigate your claim.

\section{OPEN ACCESS}




\title{
Mass bounds on light and heavy neutrinos from radiative minimal-flavor-violation leptogenesis
}

\author{
Apostolos Pilaftsis and Daniele Teresi \\ Consortium for Fundamental Physics, School of Physics and Astronomy, \\ University of Manchester, Manchester M13 9PL, United Kingdom \\ (Received 1 July 2015; published 12 October 2015)
}

\begin{abstract}
We derive novel limits on the masses of the light and heavy Majorana neutrinos by requiring successful leptogenesis in seesaw models of minimal-flavor violation (MFV). Taking properly into account radiative flavor effects and avoiding the limitations due to a no-go theorem on leptonic asymmetries, we find that the mass of the lightest of the observable neutrinos must be smaller than $\sim 0.05 \mathrm{eV}$, while the Majorana scale of lepton-number violation should be higher than $\sim 10^{12} \mathrm{GeV}$. The latter lower bound enables one to probe the existence of possible new scales of MFV, up to energies of $\sim 100 \mathrm{TeV}$, in low-energy experiments, such as $\mu \rightarrow e \gamma$ and $\mu \rightarrow e$ conversion in nuclei. Possible realizations of MFV leptogenesis in Grand Unified Theories are briefly discussed.
\end{abstract}

DOI: 10.1103/PhysRevD.92.085016

PACS numbers: $14.60 . \mathrm{St}, 11.30 . \mathrm{Hv}, 14.60 . \mathrm{Pq}, 98.80 . \mathrm{Cq}$

The hypothesis of minimal-flavor violation (MFV) [1] provides an elegant framework, even for flavor theories of new physics, to naturally implement the strong constraints from the nonobservation of sizeable flavor changing neutral currents in the quark sector. Interestingly enough, this MFV hypothesis can be extended to the lepton sector as well. In particular, one may consider seesaw models, in which all effects of lepton-flavor violation (LFV), including the observable light-neutrino masses and mixing, originate exclusively from the Yukawa interactions to three righthanded neutrinos $N_{R, \alpha}$, with $\alpha=1,2,3$, in a flavor basis with diagonal charged-lepton Yukawa couplings. Instead, the right-handed Majorana mass matrix $\boldsymbol{M}_{N}$ takes on its maximally symmetric form, $\boldsymbol{M}_{N}=m_{N} \mathbf{1}_{3}$, which means that $\boldsymbol{M}_{N}$ is invariant under $\boldsymbol{O}(3)_{N_{R}}$ rotations [2,3]. Such seesaw models allow for large hierarchies between the seesaw scale $m_{N}$ of lepton-number violation (LNV) and possible other scales of LFV, e.g. due to the soft breaking of supersymmetry [4], giving rise to observable rates for $\mu \rightarrow e \gamma, \mu \rightarrow e e e$ and $\mu \rightarrow e$ conversion in nuclei [5].

In addition, the presence of heavy Majorana neutrinos provides, via the well-established mechanism of leptogenesis [6], one of the most plausible explanations for the origin of the baryon asymmetry in the Universe (BAU). In the MFV seesaw scenario, the exact mass degeneracy of the three right-handed neutrinos leads to vanishing leptonic asymmetries [7]. However, renormalization group (RG) effects due to running from the Grand Unified Theory (GUT) scale $\mu_{X} \approx 2 \times 10^{16} \mathrm{GeV}$ to a lower heavy-neutrino scale $m_{N}$ lift the $O(3)_{N_{R}}$ symmetry in the heavy-neutrino sector, thus potentially offering the possibility to explain the BAU via the so-called mechanism of resonant leptogenesis (RL) [7,8].

In this radiative framework [9-11] of MFV, it was originally argued [10] that successful leptogenesis requires the LNV seesaw scale $m_{N}$ to be higher than $10^{12} \mathrm{GeV}$, implying sizeable neutrino Yukawa couplings larger than $\sim 0.1$, so as to fit the low-energy neutrino data. The latter would allow one to test the possible existence of additional new-physics scales mediating minimal LFV, for a wide range of energies from 1 to $100 \mathrm{TeV}$ [10], in low-energy experiments, such as the upgraded MEG experiment [12]. Subsequent elaborate studies [11], however, which include flavor effects, have suggested that $m_{N}$ could be as low as $10^{6} \mathrm{GeV}$, implying neutrino Yukawa couplings that could be as small as $10^{-4}$. Thus, these results seem to eliminate any prospects of definitely excluding the presence of new MFV scales in the near future. Most recently, however, a no-go theorem [13] for vanishing leptonic asymmetries to order $h^{4}$ in the neutrino Yukawa couplings has been proven, rendering radiative MFV leptogenesis not viable in the low$m_{N}$ region.

In this article we will show how this no-go theorem can be circumvented in a MFV framework, provided the seesaw scale $m_{N}>10^{12} \mathrm{GeV}$. The latter reopens the way of falsifying possible new leptonic MFV scales up to energies of $\sim 100 \mathrm{TeV}$. A complete treatment of flavor effects yields novel limits on the masses of the light neutrinos as well. In particular, we find that the mass of the lightest neutrino must be smaller than $\sim 0.05 \mathrm{eV}$, with the normal hierarchical light-neutrino spectrum favored over the inverted one.

In the MFV seesaw scenario, the flavor-symmetric part of the Lagrangian $\mathcal{L}_{\text {sym }}$ at the GUT scale $\mu_{X}$ reads

$$
\mathcal{L}_{\text {sym }}=\mathcal{L}_{\text {sym }}^{\mathrm{SM}}+\frac{1}{2} \bar{N}_{\mathrm{R}, \alpha}^{C}\left[M_{N}\right]^{\alpha \beta} N_{\mathrm{R}, \beta}+\text { H.c. },
$$

where $\left[M_{N}\right]^{\alpha \beta}=m_{N} \delta^{\alpha \beta}$ and $\mathcal{L}_{\text {sym }}^{\mathrm{SM}}$ is the Standard Model (SM) Lagrangian, without the neutrino and charged-lepton Yukawa interactions mediated by the couplings $h_{l}^{\alpha}$ and $y_{l}{ }^{m}$, respectively. In this work, we use the flavor-covariant 
formalism developed in Ref. [8]. Under general unitary flavor transformations $U(3)_{L} \times U(3)_{N_{R}}$, the chargedlepton SM doublets $L_{l}$ (with $l=1,2,3$ ) and the heavy neutrinos $N_{\mathrm{R}, \alpha}$ transform as

$$
\begin{gathered}
L_{l} \rightarrow L_{l}^{\prime}=V_{l}^{m} L_{m}, \\
L^{l} \equiv\left(L_{l}\right)^{\dagger} \rightarrow L^{\prime l}=V_{m}^{l} L^{m}, \\
N_{\mathrm{R}, \alpha} \rightarrow N_{\mathrm{R}, \alpha}^{\prime}=U_{\alpha}^{\beta} N_{\mathrm{R}, \beta}, \\
N_{\mathrm{R}}{ }^{\alpha} \equiv\left(N_{\mathrm{R}, \alpha}\right)^{\dagger} \rightarrow N_{\mathrm{R}}^{\prime \alpha}=U^{\alpha}{ }_{\beta} N_{\mathrm{R}}^{\beta},
\end{gathered}
$$

with the unitary transformation matrices $V_{l}^{m} \in U(3)_{L}$ and $U_{\alpha}{ }^{\beta} \in U(3)_{N_{R}}$. All the other tensors, e.g. the heavyneutrino Yukawa couplings $h_{l}^{\alpha}$, transform analogously as indicated by the position of the flavor indices.

The Lagrangian $\mathcal{L}_{\text {sym }}$ is invariant under the lepton-flavor symmetry group $G_{\mathrm{LF}}=U(3)_{L} \times U(3)_{e_{R}} \times O(3)_{N_{R}}$, where $e_{R}$ collectively denotes the three right-handed charged leptons. According to the MFV hypothesis, the flavor symmetry $G_{\mathrm{LF}}$ is explicitly broken only by the couplings $y_{l}{ }^{m}$ and $h_{l}{ }^{\alpha}$, which occur in the Lagrangian $\mathcal{L}_{\mathrm{Y}}$ of the leptonic Yukawa sector,

$$
\mathcal{L}_{\mathrm{Y}}=y_{l}{ }^{m} \bar{L}^{l} \Phi e_{R, m}+h_{l}^{\alpha} \bar{L}^{l} \tilde{\Phi} N_{\mathrm{R}, \alpha}+\text { H.c. },
$$

where $\tilde{\Phi} \equiv i \sigma_{2} \Phi^{*}$ is the Higgs hypercharge conjugate.

As mentioned above, the $O(3)$ mass degeneracy of the heavy-neutrino spectrum will be lifted by RG effects when running from the GUT scale $\mu_{X}$ to $m_{N}$. By means of the variables $t(\mu) \equiv \ln \left(\mu_{X} / \mu\right)$ and $t_{N} \equiv t\left(m_{N}\right)$, the RG equation for $\boldsymbol{M}_{N}$ is given by [14]

$$
\frac{d \boldsymbol{M}_{N}}{d t} \simeq-\frac{1}{16 \pi^{2}}\left[\left(\boldsymbol{h}^{\dagger} \boldsymbol{h}\right) \boldsymbol{M}_{N}+\boldsymbol{M}_{N}\left(\boldsymbol{h}^{T} \boldsymbol{h}^{*}\right)\right]
$$

At leading order in $\boldsymbol{h}^{\dagger} \boldsymbol{h}$, the $O(3)$-breaking part of $\boldsymbol{M}_{N}$ may be evaluated as

$$
\begin{aligned}
\Delta \boldsymbol{M}_{N}^{\mathrm{RG}} & =\left.t_{N} \frac{d \boldsymbol{M}_{N}}{d t}\right|_{t=0}+\left.\frac{t_{N}^{2}}{2} \frac{d^{2} \boldsymbol{M}_{N}}{d t^{2}}\right|_{t=0}+\cdots \\
& \simeq-\frac{m_{N}}{8 \pi^{2}} \ln \left(\frac{\mu_{X}}{m_{N}}\right) \operatorname{Re}\left(\boldsymbol{h}^{\dagger} \boldsymbol{h}\right)
\end{aligned}
$$

such that $\boldsymbol{M}_{N}$ at the energy scale $m_{N}$ relevant for RL becomes $\boldsymbol{M}_{N}\left(m_{N}\right)=m_{N} \mathbf{1}_{3}+\boldsymbol{\Delta} \boldsymbol{M}_{N}^{\mathrm{RG}}$. However, as shown in Ref. [13], all asymmetries per lepton flavor vanish through order $\boldsymbol{h}^{\dagger} \boldsymbol{h}$.

Let us briefly review the argument given in Ref. [13]. The mass matrix obtained from (5) is real and symmetric, and, as long as $\left|\left[\Delta M_{N}^{\mathrm{RG}}\right]_{\alpha \beta}\right| \ll m_{N}$, it can be diagonalized with positive eigenvalues by a real orthogonal matrix $\boldsymbol{O} \in O(3)_{N_{R}}$,

$$
\boldsymbol{M}_{N}\left(m_{N}\right)=\boldsymbol{O} \hat{\boldsymbol{M}}_{N} \boldsymbol{O}^{T},
$$

where the caret $\left({ }^{\prime}\right)$ denotes that the given quantity is evaluated in the mass eigenbasis. Since $\boldsymbol{O}$ is real, $\operatorname{Re}\left(\hat{\boldsymbol{h}}^{\dagger} \hat{\boldsymbol{h}}\right)$ is diagonal, too. Therefore, all lepton flavor asymmetries $\varepsilon_{l \alpha}$, proportional to $[8,15]$

$$
\begin{aligned}
\varepsilon_{l \alpha} & \propto \operatorname{Im}\left[\hat{h}_{l \alpha}^{*} \hat{h}_{l \beta}\left(\hat{h}^{\dagger} \hat{h}\right)_{\alpha \beta}\right]+\frac{m_{N, \alpha}}{m_{N, \beta}} \operatorname{Im}\left[\hat{h}_{l \alpha}^{*} \hat{h}_{l \beta}\left(\hat{h}^{\dagger} \hat{h}\right)_{\beta \alpha}\right] \\
& =2 \operatorname{Im}\left[\hat{h}_{l \alpha}^{*} \hat{h}_{l \beta}\right] \operatorname{Re}\left[\left(\hat{h}^{\dagger} \hat{h}\right)_{\alpha \beta}\right]+\mathcal{O}\left(h^{6}\right),
\end{aligned}
$$

with $\alpha \neq \beta$, vanish through order $h^{4}$. Notice that the inclusion of the second term in the first line of (7), often omitted in earlier studies, plays a decisive role to obtain this result. To circumvent this no-go theorem, we need to take into account the leading imaginary contribution to (5). This is contained in the second-order term $\propto t_{N}^{2}$ in the Taylor series expansion of the RG evolution of $\boldsymbol{M}_{N}$ in (5), i.e.

$$
\Delta \boldsymbol{M}_{N}^{\mathrm{RG}} \supset i t_{N}^{2} \frac{m_{N}}{\left(16 \pi^{2}\right)^{2}}\left[\operatorname{Im}\left(\boldsymbol{h}^{\dagger} \boldsymbol{h}\right), \operatorname{Re}\left(\boldsymbol{h}^{\dagger} \boldsymbol{h}\right)\right],
$$

with the commutator acting on flavor space. Notice that the so-generated imaginary term in (8) is logarithmically enhanced as compared to the leading-log higher loop-order contributions to (4). In addition, we neglect the RG effects of the Yukawa couplings in (5), as they do not produce extra imaginary terms in $\boldsymbol{\Delta} \boldsymbol{M}_{N}^{\mathrm{RG}}$, at least up to order $h^{4}$.

With the inclusion of both the contributions in (5) and (8), the matrix that diagonalizes $\boldsymbol{M}_{N}\left(m_{N}\right)$, as in (6), is now complex and unitary, thus allowing $\operatorname{Re}\left(\hat{\boldsymbol{h}}^{\dagger} \hat{\boldsymbol{h}}\right)$ to have nonzero off-diagonal elements in the mass eigenbasis. Hence, a nonzero leptonic asymmetry $\varepsilon_{l \alpha}$ can be obtained, at the expense of an additional $\mathcal{O}\left(h^{2}\right)$ suppression. This additional suppression significantly constrains the allowed region of the seesaw mass $m_{N}$ in the radiative MFV framework, contrary to previous expectations $[11,16]$. As we will see below, new correlated heavy- and light-neutrino mass bounds can be deduced by requiring successful generation of the BAU.

To fit the light-neutrino data, we write $h_{l}^{\alpha}$ in terms of an approximate parametrization similar to the one in Ref. [17]. Upon neglecting the heavy-neutrino mass splitting in the seesaw relation, the neutrino Yukawa coupling matrix $\boldsymbol{h}$ may be determined as

$$
\boldsymbol{h} \simeq-i \frac{\sqrt{2 m_{N}}}{v} \boldsymbol{U}_{\mathrm{PMNS}} \hat{\boldsymbol{m}}_{\nu}^{\frac{1}{2}} \boldsymbol{R},
$$

where $v=246 \mathrm{GeV}, \boldsymbol{U}_{\text {PMNS }}$ is the Pontecorvo-MakiNakagawa-Sakata (PMNS) matrix [18], $\hat{\boldsymbol{m}}_{\nu}$ is the diagonal matrix of light-neutrino masses, and $\boldsymbol{R}$ is a complex orthogonal matrix that can be expressed in terms of three complex Euler angles $\psi_{1,2,3}$. We then calculate the heavyneutrino mass matrix $\boldsymbol{M}_{N}\left(m_{N}\right)$ by using the RG running 
given in (5) and (8). We diagonalize $\boldsymbol{M}_{N}\left(m_{N}\right)$ by means of a 3-by-3 unitary matrix $\boldsymbol{U}$ [which generalizes $\boldsymbol{O}$ in (6)] and rotate the Yukawa coupling matrix $\boldsymbol{h}$ to the mass eigenbasis. We stress here that this last point, missed out in several earlier studies, becomes of utmost importance in our analysis, in light of the no-go theorem outlined above.

We calculate the lepton asymmetry generated via the RL mechanism by means of the flavor-covariant rate equations developed in Ref. [8]. To simplify our numerical analysis, we treat heuristically the effect of regenerative $C P$ violation via oscillations in medium $[8,19]$, due to heavy-neutrino off-diagonal number densities (which quantify the quantum coherences between different flavors), by including a factor of 2 enhancement with respect to the contribution of the heavy-neutrino mixing alone. The latter is obtained including only the diagonal heavy-neutrino number densities in the mass eigenbasis. In the radiative MFV scenario under study, this is a rather good approximation [8], since one is mostly in the weakly resonant regime in which $\Gamma_{N_{\alpha}} \ll \mid m_{N_{\alpha}}-$ $m_{N_{\beta}} \mid \ll m_{N}$, where $\Gamma_{N_{\alpha}}$ is the width of $N_{R, \alpha}$. As we will see, successful leptogenesis is possible only for $m_{N}>10^{12} \mathrm{GeV}$, and thus it is safe to neglect the decoherence effects due to the SM Yukawa couplings, which are out of equilibrium in this kinematic region. However, in spite of this simplification, an unflavored treatment as usually followed in high-scale hierarchical leptogenesis would be inappropriate because the three heavy neutrinos decay, in general, into three different superpositions of charged leptons.

With these simplifications, and assuming that the heavyneutrino evolution is in the strong-washout regime, i.e. that $\Gamma_{N_{\alpha}}$ is much larger than the Hubble rate $H\left(T=m_{N}\right)$, the equation for the lepton asymmetry due to mixing $\delta \boldsymbol{\eta}_{\text {mix }}^{L}$ can be written, in matrix form, as [8]

$$
\frac{d}{d z} \boldsymbol{\delta} \boldsymbol{\eta}_{\text {mix }}^{L}=\frac{z^{3} K_{1}(z)}{2}\left(\frac{\boldsymbol{\varepsilon}}{z}-\frac{1}{3}\left\{\boldsymbol{\delta} \boldsymbol{\eta}_{\text {mix }}^{L}, \mathbf{K}^{\mathrm{eff}}\right\}\right),
$$

where $z=m_{N} / T$, with $T$ being the temperature, and $K_{1}(z)$ is the modified Bessel function. $\boldsymbol{\varepsilon}$ and $\mathbf{K}^{\text {eff }}$ are 3-by-3 matrices, in charged-lepton flavor space, of leptonic flavor asymmetries and K-factors, respectively. Their explicit form can be found in Ref. [8]. The former describes the $C P$ asymmetry in the heavy-neutrino decays $N \rightarrow L \Phi$ due to flavor mixing, whereas the latter describes the washout of the lepton asymmetry due to inverse decays and scattering processes.

It is convenient to rotate (10) to the eigenbasis of $\mathbf{K}^{\text {eff }}$, denoted by a tilde ( $)$, where the evolution of the diagonal entries $\left[\delta \tilde{\eta}_{\text {mix }}^{L}\right]_{l l}$ decouples from the respective off-diagonal ones. This allows one to solve (10) semianalytically, assuming vanishing initial asymmetry, obtaining the final asymmetry $\delta \eta^{L}$ as
TABLE I. Region scanned in the numerical analysis. All the parameters are generated randomly, with uniform distributions in the intervals quoted.

\begin{tabular}{lcc}
\hline \hline Parameter & Lower limit & Upper limit \\
\hline $\log _{10}\left(m_{N} / \mathrm{GeV}\right)$ & $11 \log _{10}(5)$ & 15 \\
$\min m_{\nu_{l}} / \mathrm{eV}$ & 0 & 0.06 \\
$\delta, \phi_{1,2}, \operatorname{Re} \psi_{1,2,3}$ & 0 & $2 \pi$ \\
$\operatorname{Im} \psi_{1,2,3}$ & -3 & 3 \\
\hline \hline
\end{tabular}

$$
\delta \eta^{L} \approx 2 \times \delta \eta_{\mathrm{mix}}^{L}=2 \sum_{l} \tilde{\varepsilon}_{l l} \kappa_{\mathrm{fin}}\left(\tilde{\mathrm{K}}_{l l}^{\mathrm{eff}}\right) .
$$

The efficiency factor $\kappa_{\text {fin }}$ can be obtained by first integrating numerically the differential equation

$$
\frac{d}{d z} \kappa=\frac{z^{3} K_{1}(z)}{2}\left(\frac{1}{z}-\frac{2}{3} \tilde{\mathrm{K}}_{l l}^{\mathrm{eff}} \kappa\right)
$$

and then calculating the final stationary limit $\kappa_{\text {fin }}=\kappa(z \rightarrow \infty)$. In the leptonic strong-washout regime $\mathrm{K}_{L}^{\min } \equiv \min _{l} \tilde{\mathrm{K}}_{l l}^{\text {eff }} \gg 1$, the final asymmetry does not depend on its initial value, and $\kappa_{\text {fin }} \simeq 3 /\left(2 \tilde{\mathrm{K}}_{l l}^{\text {eff }}\right)$. In the opposite
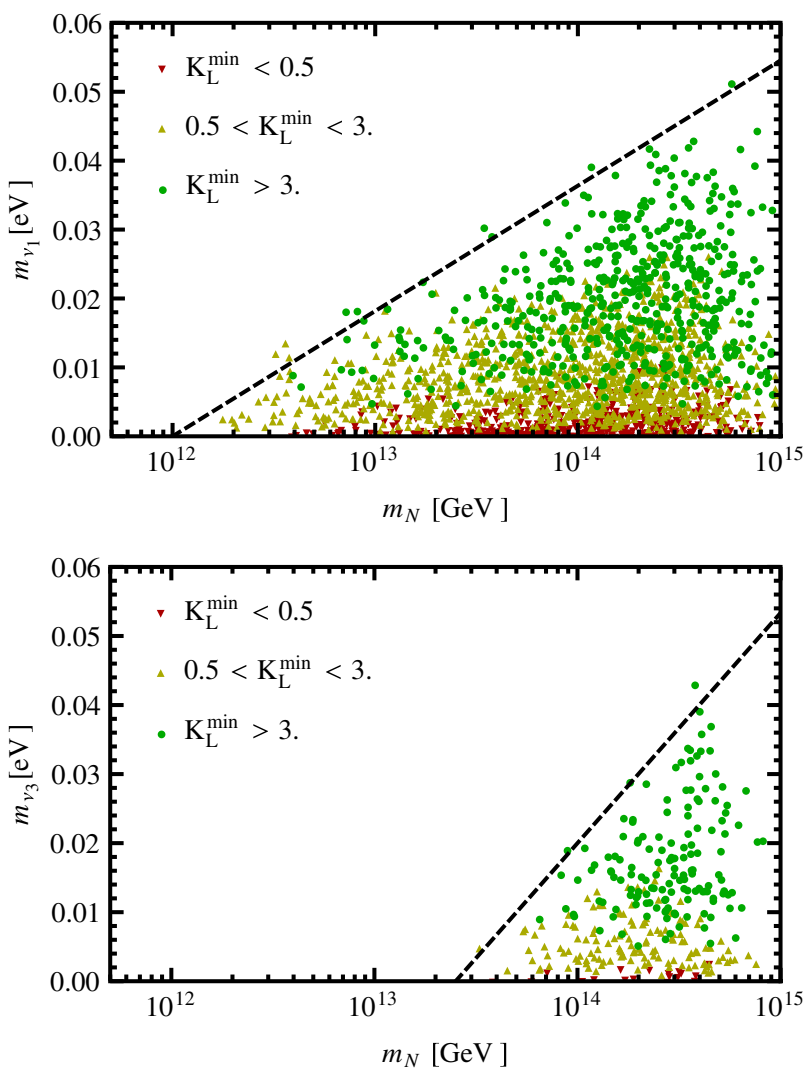

FIG. 1 (color online). Scatter plots of the values of the heavyand lightest-neutrino masses that allow successful leptogenesis, assuming normal (top panel) and inverted (bottom panel) hierarchy. The dashed lines denote the approximate bounds given in (13). 

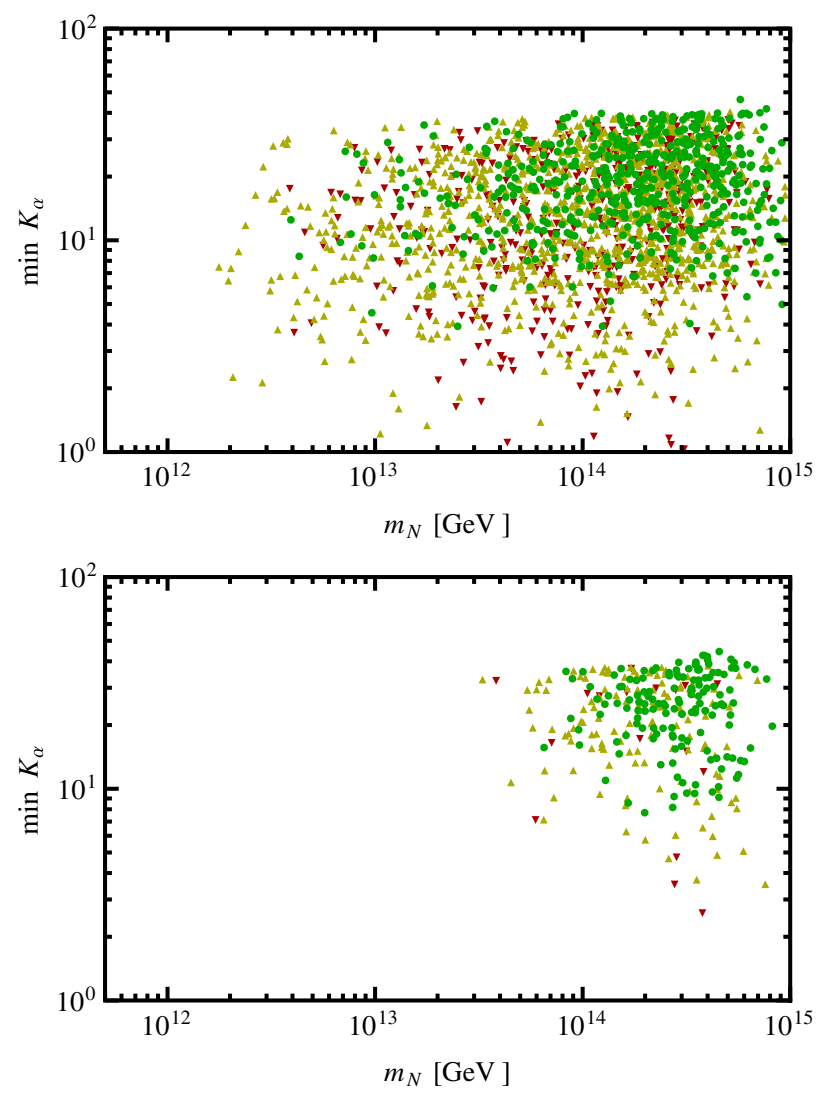

FIG. 2 (color online). Smallest heavy-neutrino K-factor, as function of their mass, for the numerical scan with normal (top panel) and inverted (bottom panel) hierarchy. Legend as in Fig. 1.

leptonic weak-washout limit, one has $\kappa_{\text {fin }} \simeq 1$, but a preexisting asymmetry is not washed out.

To study the constraints derived from successful leptogenesis in the radiative MFV scenario, we perform a numerical scan of the parameter space. For both normal $(\mathrm{NH})$ and inverted hierarchy $(\mathrm{IH})$ of the light neutrinos, we generate a set of $2.5 \times 10^{5}$ random points. The parameters of the scan are the singlet seesaw mass scale $m_{N}$, the mass of the lightest neutrino $m_{\nu_{1}}\left(m_{\nu_{3}}\right)$ in the NH (IH) case, the Dirac phase $\delta$ and Majorana phases $\phi_{1,2}$ of the PMNS matrix and the complex Euler angles $\psi_{1,2,3}$ appearing in the matrix $\boldsymbol{R}$ in (9). The limits of the scanned regions are exhibited in Table I. We have checked that enlarging the scanned region does not affect significantly the results presented below. In addition, we impose the following cuts: (i) the perturbativity condition $\left|\hat{h}_{l \alpha}\right|<1$ and (ii) $\hat{\mathrm{K}}_{\alpha}>1$, where $\hat{\mathrm{K}}_{\alpha} \equiv \Gamma_{N_{\alpha}} /\left(\zeta(3) H\left(t=m_{N}\right)\right)$ are the heavy-neutrino washout factors.

In Fig. 1 we plot the scanned points that entail successful leptogenesis $\left|\delta \eta^{L}\right|>\left|\delta \eta_{\mathrm{obs}}^{L}\right|=2.47 \times 10^{-8}$ [8], in terms of the heavy-neutrino seesaw mass $m_{N}$, and the lightest neutrino masses $m_{\nu_{1,3}}$. We find that a $\mathrm{NH}$ spectrum for the light neutrinos is more pronounced, with a larger allowed parameter space, over an IH one. Moreover, requiring successful leptogenesis yields the following
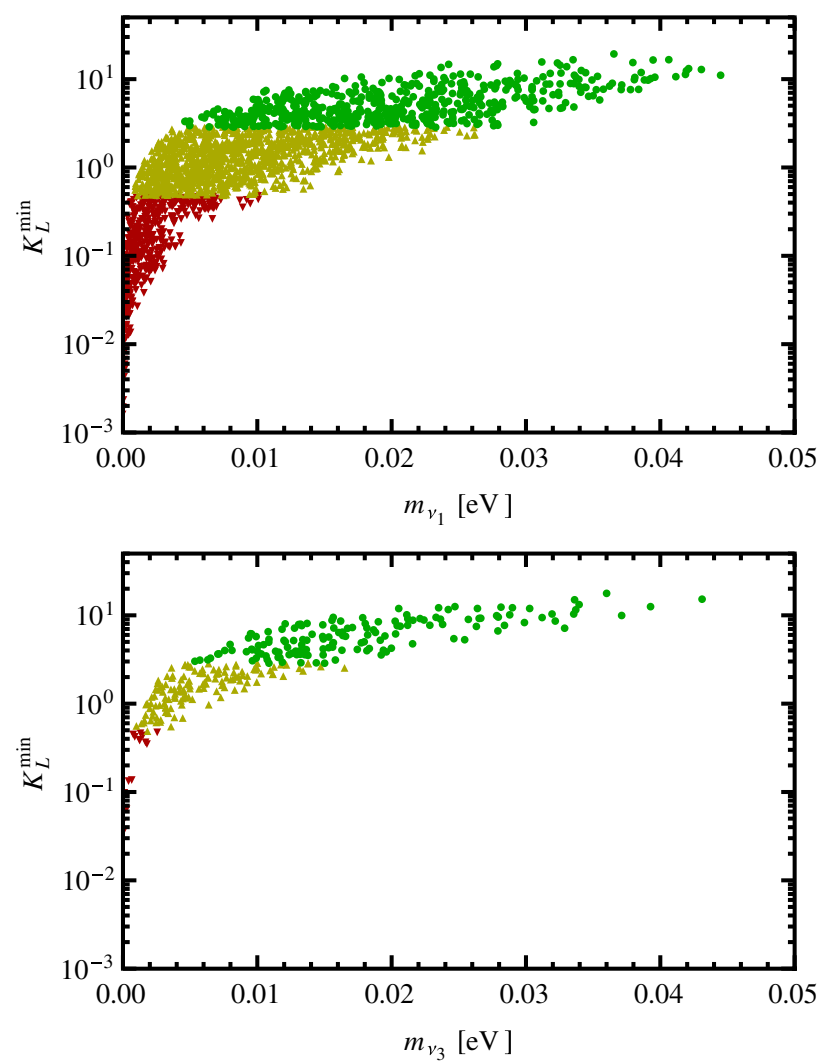

FIG. 3 (color online). Smallest effective charged-lepton Kfactor $\mathrm{K}_{L}^{\mathrm{min}}$, as a function of the lightest-neutrino mass, for the numerical scan with normal (top panel) and inverted (bottom panel) hierarchy. Legend as in Fig. 1.

approximate combined bounds on the heavy- and lightneutrino masses:

$$
\begin{aligned}
& \log _{10} \frac{m_{N}}{10^{12} \mathrm{GeV}}>55 \frac{m_{\nu_{1}}}{\mathrm{eV}} \quad(\mathrm{NH}), \\
& \log _{10} \frac{m_{N}}{10^{12} \mathrm{GeV}}>1.4+30 \frac{m_{\nu_{3}}}{\mathrm{eV}} \quad(\mathrm{IH}) .
\end{aligned}
$$

In particular, we obtain the approximate absolute bounds:

$$
m_{N}>10^{12} \mathrm{GeV}, \quad \min m_{\nu_{l}}<0.05 \mathrm{eV} .
$$

Observe that the light-neutrino mass bound is compatible (at the $1.9 \sigma$ confidence level) with the recent evidence for a neutrino mass scale $\sum m_{\nu_{l}}=(0.320 \pm 0.081) \mathrm{eV}$, obtained from cosmic microwave background (CMB) and lensing observations [20].

In the rightmost part of the plots in Fig. 1, i.e. for $m_{N} \sim 10^{15} \mathrm{GeV}$, the perturbativity cut $\left|\hat{h}_{l \alpha}\right|<1$ gets saturated, and the error in the approximate seesaw-inversion parametrization (9) reaches upper values of 5\%-10\%. Also, in this region of the parameter space, the weakly resonant condition is satisfied only mildly. Therefore, we expect our results to be less accurate for $m_{n} \gtrsim 10^{15} \mathrm{GeV}$. On the other hand, in Fig. 2 we plot the successful points in terms of the 
smallest heavy-neutrino K-factor. We see that the heavyneutrino strong-washout requirement is comfortably satisfied in all the parameter space. This justifies the simplified form of the rate equation (10), for which quantum memory effects are omitted. Finally, in Fig. 3 we show the dependence of the smallest leptonic K-factor on the mass of the lightest neutrino. We see that most points have moderate and large values of leptonic $\mathrm{K}$-factors in the parameter space of successful leptogenesis (see also Fig. 1). Thus, our predictions for the BAU will be largely independent of moderate preexisting leptonic asymmetries in the MFV framework. Requiring large leptonic washout factors $\mathrm{K}_{L}^{\mathrm{min}}>3$ leads to an additional approximate lower bound on the light neutrino masses $\min m_{\nu_{l}}>0.004 \mathrm{eV}$.

The requirement of successful MFV leptogenesis restricts the seesaw scale $m_{N}$ of LNV to be above $10^{12} \mathrm{GeV}$, which is much closer to the GUT scale. It is therefore natural to investigate whether GUT embeddings of the MFV hypothesis are feasible. Indeed, a possible MFV GUT based on $S U(5)$ has already been suggested in Ref. [21], but it would be interesting to explore further this possibility, specifically within the context of $S O(10)$ unification. In the latter case, a minimal Higgs content for the generation of fermion masses, with a 10- and a 126-dimensional Higgs representations [22], cannot accommodate both degenerate $N_{R, \alpha}$ and nontrivial mixing matrices [23]. Nevertheless, it appears possible to construct MFV $S O(10)$ theories by considering the full allowed Yukawa sector, which includes also a complex 120-dimensional Higgs field. Detailed studies of such scenarios are beyond the scope of this article and may be given elsewhere.

The work of A.P. is supported by the LancasterManchester-Sheffield Consortium for Fundamental Physics under STFC Grant No. ST/L000520/1.
[1] S. L. Glashow, J. Iliopoulos, and L. Maiani, Phys. Rev. D 2 , 1285 (1970); A. J. Buras, P. Gambino, M. Gorbahn, S. Jager, and L. Silvestrini, Phys. Lett. B 500, 161 (2001); G. D'Ambrosio, G. F. Giudice, G. Isidori, and A. Strumia, Nucl. Phys. B645, 155 (2002).

[2] A. Pilaftsis and T.E. J. Underwood, Phys. Rev. D 72, 113001 (2005).

[3] V. Cirigliano, B. Grinstein, G. Isidori, and M. B. Wise, Nucl. Phys. B728, 121 (2005).

[4] F. Borzumati and A. Masiero, Phys. Rev. Lett. 57, 961 (1986).

[5] A. Ilakovac, A. Pilaftsis, and L. Popov, Phys. Rev. D 87, 053014 (2013).

[6] M. Fukugita and T. Yanagida, Phys. Lett. B 174, 45 (1986).

[7] A. Pilaftsis, Phys. Rev. D 56, 5431 (1997); A. Pilaftsis and T. E. Underwood, Nucl. Phys. B692, 303 (2004).

[8] P. Bhupal Dev, P. Millington, A. Pilaftsis, and D. Teresi, Nucl. Phys. B886, 569 (2014).

[9] R. Gonzalez Felipe, F. R. Joaquim, and B. M. Nobre, Phys. Rev. D 70, 085009 (2004); K. Turzynski, Phys. Lett. B 589, 135 (2004); G. C. Branco, R. Gonzalez Felipe, F. R. Joaquim, and B. M. Nobre, Phys. Lett. B 633, 336 (2006).

[10] V. Cirigliano, G. Isidori, and V. Porretti, Nucl. Phys. B763, 228 (2007).

[11] G. C. Branco, A. J. Buras, S. Jager, S. Uhlig, and A. Weiler, J. High Energy Phys. 09 (2007) 004; S. Uhlig, J. High Energy Phys. 11 (2007) 066; V. Cirigliano, A. De Simone,
G. Isidori, I. Masina, and A. Riotto, J. Cosmol. Astropart. Phys. 01 (2008) 004.

[12] A. M. Baldini et al., arXiv:1301.7225.

[13] P. S. B. Dev, P. Millington, A. Pilaftsis, and D. Teresi, Nucl. Phys. B897, 749 (2015).

[14] P. H. Chankowski and Z. Pluciennik, Phys. Lett. B 316, 312 (1993); K. Babu, C. N. Leung, and J. T. Pantaleone, Phys. Lett. B 319, 191 (1993).

[15] L. Covi, E. Roulet, and F. Vissani, Phys. Lett. B 384, 169 (1996).

[16] F. F. Deppisch and A. Pilaftsis, Phys. Rev. D 83, 076007 (2011).

[17] J. Casas and A. Ibarra, Nucl. Phys. B618, 171 (2001).

[18] We use the best-fit values for the light-neutrino oscillation parameters from F. Capozzi, G. L. Fogli, E. Lisi, A. Marrone, D. Montanino, and A. Palazzo, Phys. Rev. D 89, 093018 (2014). The Dirac and Majorana phases are left unconstrained.

[19] P. Bhupal Dev, P. Millington, A. Pilaftsis, and D. Teresi, Nucl. Phys. B891, 128 (2015).

[20] R. A. Battye and A. Moss, Phys. Rev. Lett. 112, 051303 (2014).

[21] B. Grinstein, V. Cirigliano, G. Isidori, and M. B. Wise, Nucl. Phys. B763, 35 (2007).

[22] K. S. Babu and R. N. Mohapatra, Phys. Rev. Lett. 70, 2845 (1993).

[23] For a recent analysis, see A. Dueck and W. Rodejohann, J. High Energy Phys. 09 (2013) 024 and references therein. 